\title{
Local Thermodynamic Equilibrium at Three Levels
}

\author{
by Geoffrey L. Sewell \\ Department of Physics, Queen Mary University of London \\ (e-mail: g.l.sewell@qmul.ac.uk) \\ Mile End Road, London E1 4NS, UK
}

\begin{abstract}
We present coordinated formulations of local thermodynamical equilibrium conditions at three levels, namely the macroscopic one of classical thermodynamics, the mesoscopic one of hydrodynamical fluctuations and the microscopic one of quantum statistical mechanics. These conditions are all expressed in terms of the hydrodynamical variables of the macroscopic picture, and the quantum statistical ones are shown to imply a local version of the zeroth law.
\end{abstract}

Keywords: local equilibrium, macro-, meso- and micro-scopic pictures, operator algebras, thermodynamic completeness, zeroth law 


\section{Introduction}

The concept of local thermodynamic equilibrium (LTE) is basic, at the macroscopic level, to nonequilibrium thermodynamics [DM, LL]; while at the microscopic level, it features in certain statistical mechanical models, such as the quantal ones of [Da, NY] and the classical ones of [KMP, DIPP, Pr]. However, there does not appear to be a general, coherent formulation of this concept that coordinates the pictures of LTE at the macroscopic level of classical nonequilibrium thermodynamics, the mesoscopic one of hydrodynamical fluctuations and the microscopic one of quantum statistical mechanics. The object of this note is to present such a formulation and to show that its quantum statistical component leads to a local version of the zeroth law. This objective marks part of our programme [Se1-3] of building bridges between the quantum microscopic and classical macroscopic pictures of matter, rather than an attempted derivation of the latter from the former.

We base our treatment on the generic model of a nonrelativistic many-particle quantum system, $\Sigma$, with translationally invariant interactions, which occupies an open, connected region of a $d$-dimensional Euclidean space, $X$, and is coupled at its boundary to an array, $\mathcal{R}$, of reservoirs. We assume, in a standard way [Ca], that its equilibrium thermodynamics is based on the form of an entropy function, $S$, of a set $Q\left(=\left(Q_{1}, \ldots, Q_{n}\right)\right)$ of extensive conserved variables, of which $Q_{1}$ is the energy. Assuming that $S(Q)$ is also extensive, its density is a volume independent function, $s$, of $q$, the density of $Q$. This latter density represents the state of $\Sigma$ at the thermodynamic level.

For the nonequilibrium situation, we assume that the classical continuum mechanics of the model is given by an autonomous evolution of the position and time dependent density, $q(x, t)$, of $Q$, which takes the form*

$$
\frac{\partial}{\partial t} q(x, t)=\Phi(q: x, t),
$$

where $\Phi$ is a functional of $q$. We assume, for simplicity, that this equation is invariant under space-time scale trransformations of the form $x \rightarrow \lambda x, t \rightarrow \lambda^{c} t$, where $\lambda$ is a variable positive parameter and $c$ is a positive constant. This assumption is satisfied in the cases of nonlinear diffusion and inviscid Eulerian hydrodynamics. In the former case, $\Phi(q)$ takes the form $\nabla \cdot(K(q) \nabla q)$, with $K$ a positive $n$-by-n matrix valued function of $q$ and $c=2$ : in the latter case, it is easily seen from the Euler equations that $c=1$. On the other hand, the assumption is not satisfied by Navier-Stokes equations for viscous flow: this case requires a modified treatment [Se3] that we shall briefly discuss in Section 5 .

In order to treat $\Sigma$ on different levels of macroscopicality, we need to specify the relationships between the length and time scales for those levels. Our choice is to take the unit of length for the macroscopic and mesoscopic pictures to be $L$ times that of the microscopic one, where $L=N^{1 / d}, N$ being the number of particles in $\Sigma$.Thus, denoting by $\Omega$ the region of $X$ occupied by $\Sigma$ on the macroscopic scale, this same region is $\Omega_{L}=$

* This assumption is manifestly satisfied by diffusion and heat conduction processes, even nonlinear ones, as well as by Navier-Stokes hydrodynamics. 
$L \Omega(=\{L x \mid x \in \Omega\})$ on the microscopic one. We take $\Omega$ to be $L$-independent and choose the macroscopic units so that its volume is unity: the volume of $\Omega_{L}$ is therefore $L^{d}=N$.

In view of the assumed scale invariance of the macroscopic dynamics of the model, specified following Eq. (1.1), the choice of $L$ for the ratio of the macroscopic unit of length to the microscopic one demands that the corresponding ratio for the units of time is $L^{c}$. Hence the time scales for the microscopic and macroscopic pictures are infinitely separated in the hydrodynamic limit $L \rightarrow \infty$. The system therefore supports dynamics over two quite different time scales, namely a macroscopic one for which we continue to denote the time variable by $t$ and a microscopic one for which we denote that variable by $\tau$. Indeed, the macroscopic time may be considered to be 'frozen' at a value $t$ while the system undergoes its quantum evolution on the microscopic time scale. Hence, it is natural to anticipate that a submacroscopic* region of the system concentrated around a point $x$ of $\Omega$ is driven by its microscopic kinetics to a state of equilibrium corresponding to the value of $q(x, t)$. This is conceived, heuristically, to be the essential mechanism whereby local equilibrium is generated at all levels.

In addition to $L$, other key parameters of the model are the Planck and Boltzmann constants, $\hbar$ and $k$. We assume that, in the units of the macroscopic picture, these two quantities are extremely small**. Accordingly, we employ limits where $\hbar$ and $k$, as well as $L^{-1}$, tend to zero in certain parts of our treatment. Specifically, the limit $\hbar \rightarrow 0$ is implicit in the assumption of macroscopic classicality that underlies the phenomenological picture and which we extend to the mesoscopic one of hydrodynamical fluctuations***; the limit $L \rightarrow \infty$ is standard for the connection between microscopic and hydrodynamic properties of matter; and the limit $k \rightarrow 0$, which we term the Boltzmann limit, arises in our formulation of hydrodynamic fluctuations, which for equilibrium states are governed by Einstein's relation, $P=$ const $\exp (S / k)$, between their probability distribution, $P$, and the entropy $S$.

We begin our treatment in Section 2 by formulating largely standard macroscopic, mesoscopic and microscopic pictures of global thermal equilibrium of the model in terms that facilitate a subsequent passage to corresponding pictures of LTE. For the macroscopic description, in Section 2.1, we express the thermodynamics of the model in terms of the entropy density $s(q)$ and its Legendre transform ${ }^{* * * *}, \pi(\theta)$, where $\theta$, the thermodynamic conjugate of $q$, is just the first derivative of the $s(q)$. We term it the control variable. For the mesoscopic picture, in Section 2.2, we employ Einstein's formula, in the Boltzmann limit, to express the statistical properties of the hydrodynamical fluctuations in terms of the Hessian of $\pi(\theta)$. For the microscopic picture, in Section 2.3, we formulate the model in

* Here a submacroscopic region is one that is extremely small in macroscopic terms but still large enough to contain an enormous number of particles.

** For example, in SI units, $\hbar$ and $k$ are of the order of $10^{-34}$ and $10^{-23}$ respectively.

*** This supplementary assumption is consistent with the known result [GVV] that the fluctuation fields satisfy commutation relations, which are applicable to both classical and bosonic fields

**** This is just the ratio of the pressure to the temperature. 
operator algebraic terms, characterising its equilibrium states by a global thermodynamical stability (GTS) condition that corresponds precisely to that of the macroscopic description. We then employ the quantum statistical model to obtain a rather simple condition for the thermodynamical completeness of the variables $Q$ of the macroscopic picture. Furthermore we recall that the GTS condition implies the dynamical one of Kubo-Martin-Schwinger (KMS), subject to certain technical assumptions; and consequently [KFGV] that the model conforms to the zeroth law, in that it drives finite systems to which it is locally coupled to equilibrium at its own temperature.

In Section 3 we pass from global to local equilibrium conditions in the following way. We start, in Section 3.1, by recalling that the equation of motion (1.1) involves the assumption that, at a local level, the system supports a thermodynamics in which the entropy density at position $x$ and time $t$ is just the equilibrium entropy density function, $s$, of $q(x, t)$, the local density of $Q$. This is the local equilibrium hypothesis at the macroscopic level and $q(x, t)$ is now the solution of Eq. (1.1), subject to the boundary conditions imposed by the reservoirs $\mathcal{R}$. The thermodynamical conjugates $\theta(x, t)$ of $q(x, t)$ and $\pi$ of $s$ are then obtained by Legendre transformation, just as for global equilibrium. In Section 3.2 , we recall that the equilibrium condition for the hydrodynamical fluctuation process, formulated in Section 2. reduces locally to a very simple form, expressed in terms of the Hessian of $\pi(\theta)$. Accordingly, we assume that, for the nonequilibrium situation, the local equilibrium condition takes the same form, with the constant $\theta$ replaced by $\theta(x, t)$. In Section 3.3, we consider the quantum statistical picture of the system in a region that, in the macroscopic units, is an open neighbourhood $\mathcal{N}(\epsilon, x)$ of $x(\in \Omega)$, that shrinks to the point $x$ as $\epsilon \rightarrow 0$. Since this region is $\mathcal{N}_{L}(\epsilon, x):=L \mathcal{N}(\epsilon, x)$, when viewed on the microscopic scale, it becomes infinitely large there, covering the whole of $X$, as $L \rightarrow \infty$. Hence, by employing limits in which first $L \rightarrow \infty$ and then $\epsilon \rightarrow 0$, we achieve a description in which the neighbourhood corresponds to a hydrodynamic point in being macroscopically small and microscopically large. In this limiting situation, the quantum model of $\Sigma$, as restricted to this region, reduces to that of an infinite system, and the local equilibrium condition is simply that of GTS, and hence of KMS, corresponding to the value $\theta(x, t)$ of the control variable. Thus we arrive at the picture wherein the local equilibrium conditions at all levels are determined by the macroscopic one, in that their precise forms are just those of global equilibrium, but with the constant valued $\theta$ replaced by the position and time dependent $\theta(x, t)$.

In Section 4, we show, by an adaptation of the argument of [KFGV], that the LTE condition at the quantum statistical level implies a local version of the zeroth law in that, in the limit $L \rightarrow \infty$, its coupling to a finite system $\Gamma$ in a neighbourhood of $x$ drives the latter to equilibrium at the local temperature of $\Sigma$.

We conclude in Section 5 with a summary of our results and a sketch of how they may be extended to cases such as that of Navier-Stokes fluids, where the macroscopic evolution is not scale invariant.

\section{Equilibrium Thermodynamics}


We formulate the equilibrium thermodynamics of $\Sigma$ here on macroscopic, mesoscopic and microscopic levels.

\subsection{The Macroscopic Picture}

In a standard formulation [Ca], the thermodynamics of $\Sigma$ may be expressed in terms of a set of extensive conserved variables $Q=\left(Q_{1}, \ldots, Q_{n}\right)$ and an entropy function, $S$, of these: in particular, $Q_{1}$ is the energy of the system. We assume that the range of $Q$ is a convex subset of $\mathbf{R}^{n}$ and we note that the demand of thermodynamic stability ensures that the function $S$ is concave. Further, its value, $S(Q)$, like that of $Q$, is extensive. We shall provide a quantum statistically based thermodynamic completeness condition for $Q$ in Section 3.3.

The fundamental thermodynamic formula for the change in $S$ incurred in quasi- static transitions between equilibrium states is

$$
T d S=d E+\Sigma_{j=2}^{n} f_{j} d Q_{j}+p d V
$$

where $T$ is the temperature, $p$ the pressure, $V$ the volume of $\Omega$ and $f_{j} d Q_{j}$ is the work done by the system in effecting an infinitesimal change in $Q_{j}$. In view of the extensivity of $Q$ and $S(Q)$, these may be expressed as $q V$ and $s(q) V$, respectively, where the function $s$ is $V$-independent and, like $S$, is concave. Here $q$ is an $n$-tuple $\left(q_{1}, \ldots, q_{n}\right)$ and represents the thermodynamic state of $\Sigma$. We denote its range by $\mathcal{Q}$, and we shall sometimes denote $q_{1}$, the energy density, by $e$.

Since $Q=q V$ and $S(Q)=s(q) V$, Eq. (2.1.1) is equivalent to the formula

$$
\left[T d s-d e-\sum_{j=2}^{n} f_{j} d q_{j}\right] V+\left[T s-e-\sum_{j=2}^{n} f_{j} q_{j}-p\right] d V=0
$$

from which it follows that

$$
T d s=d e+\sum_{j=2}^{n} f_{j} d q_{j}
$$

and

$$
p=T s-e-\sum_{j=2}^{n} f_{j} q_{j}
$$

Note that these formulae and their consequences pertain to the thermodynamic limit of the corresponding statistical mechanical ones, since the extensivity relations $Q=q V$ and $S(Q)=s(q) V$ represent approximations wherein surface effects are neglected.

The thermodynamic conjugate of $q$ is the control variable, $\theta$, defined by the formula

$$
\theta=\left(\theta_{1}, \ldots, \theta_{n}\right)=s^{\prime}(q):=\left(\partial s(q) / \partial q_{1}, \ldots, \partial s(q) / \partial q_{n}\right)
$$

Hence, by Eqs. (2.1.2) and (2.1.4),

$$
\theta_{1}=T^{-1} \text { and } \theta_{j}=T^{-1} f_{j} \text { for } j=2, ., n .
$$


We denote the range of $\theta$ by $\Theta$, which we term the control space and assume to be convex.

The thermodynamic potential conjugate to $s$ is defined to be the function, $\pi$, on $\Theta$ given by the formula

$$
\pi(\theta)=\sup _{q^{\prime} \in \mathcal{Q}}\left(s\left(q^{\prime}\right)-\theta \cdot q^{\prime}\right),
$$

where the dot denotes the $\mathbf{R}^{n}$ inner product. Since $s$ is concave, it follows from the definition of $\Theta$ that the supremum of this formula is attained by a value, $q$, of $q^{\prime}$ related to $\theta$ by Eq. (2.1.5), i.e.

$$
\pi(\theta)=s(q)-\theta \cdot q
$$

We shall presently discuss conditions under which this $q$ is unique. In any case, it follows from Eqs. (2.1.3), (2.1.5) and (2.1.7) that

$$
\pi(\theta)=p / T
$$

In view of this formula, we term $\pi$ the reduced pressure. It follows from Eq. (2.1.6) that $\pi$ is convex. Hence, in a standard terminology for convex functions, a tangent to the graph of $\pi$ at a point $P$ is just a line through $P$ that lies below that graph. Thus, the set, $\mathcal{T}_{\theta}$, of tangents at the point $(\theta, \pi(\theta))$ consists of the lines through that point whose slopes, $r$, satisfy the inequality

$$
\pi\left(\theta^{\prime}\right)-\pi(\theta) \geq r .\left(\theta^{\prime}-\theta\right) \forall \theta^{\prime} \in \Theta .
$$

It follows immediately from this formula that $\mathcal{T}_{\theta}$ is convex. We denote the set of its extremal elements by $\mathcal{E}\left(\mathcal{T}_{\theta}\right)$. Further, by Eqs. (2.1.6) and (2.1.7),

$$
\pi\left(\theta^{\prime}\right)-\pi(\theta) \geq-q \cdot\left(\theta^{\prime}-\theta\right) \forall \theta^{\prime} \in \Theta,
$$

which signifies, by Eq. (2.1.9), that $-q \in \mathcal{T}_{\theta}$. Thus, the equilibrium states, $q$, corresponding to the control parameter $\theta$ are elements of $-\mathcal{T}_{\theta}:=\left\{-r \mid r \in \mathcal{T}_{\theta}\right\}$. Conversely [Se1, Prop. 6.3.1], assuming that this set is contained in $\mathcal{Q}$, it comprises all the equilibrium states, as defined by Eq. (2.1.7). Hence we identify the equilibrium states with the elements of $-\mathcal{T}_{\theta}$. In particular, we assume* that the pure phases are represented by the extremals, $-\mathcal{E}\left(\mathcal{T}_{\theta}\right)$. Thus, in the case where $\pi$ is differentiable at the value $\theta$ of the control parameter, $\mathcal{T}_{\theta}$ consists of the single element, $-q$, corresponding to a pure phase and defined by the formula

$$
\pi^{\prime}(\theta)=-q .
$$

It follows from this equation and Eq. (2.1.4) that the correspondence between $q$ and $\theta$ is one-to-one and therefore that, in the pure phase regime, we may equivalently represent the state of $\Sigma$ by either of these variables. Moreover, assuming that, in this regime, both $s$ and $\pi$ are twice differentiable, it follows from Eqs. (2.1.4) and (2.1.11) that the Hessians, $s^{\prime \prime}(q)\left(:=\left[\partial^{2} s(q) / \partial q_{j} \partial q_{k}\right]\right)$ and $\pi^{\prime \prime}(\theta)\left(:=\left[\partial^{2} \pi(\theta) / \partial \theta_{j} \partial \theta_{k}\right]\right)$, are related by the equation

$$
\pi^{\prime \prime}(\theta) s^{\prime \prime}(q)=s^{\prime \prime}(\bar{q}) \pi^{\prime \prime}(\theta)=-I,
$$

* This assumption is supported in Section 2.3 on the quantum statistical grounds that the extremals are the only equilibrium states for which $q$ is sharply defined (cf. [Ru]): for the others this is a random variable with non-zero dispersion. 
i.e.

$$
\pi^{\prime \prime}(\theta)=-\left[s^{\prime \prime}(q)\right]^{-1} .
$$

\subsection{The Mesoscopic Picture: Equilibrium Fluctuations}

Here we employ the same spatial scale as for the macroscopic picture, though now the density $q$ of $Q$ is a function of position. The statistics of the equilibrium fluctuations are governed by the canonical modification of Einstein's formula for the situation where the control variable $\theta$ is maintained by the reservoirs at the constant value $\bar{\theta}$. Assuming that the system is in a pure phase, the corresponding value, $\bar{q}$, of $q$ is $-\pi^{\prime}(\bar{\theta})$, by Eq. (2.1.11). The probability distribution, $P$, for the macroscopic variables is then given formally by the equation

$$
P=\text { const.exp }([S-\bar{\theta} \cdot Q] / k),
$$

i.e.

$$
P=\text { const.exp }\left(k^{-1} \int_{\Omega} d x[s(q(x))-\bar{\theta} \cdot q(x)]\right)
$$

or, equivalently,

$$
P=\text { const.exp }\left(k^{-1} \int_{\Omega} d x[s(q(x))-\bar{\theta} \cdot(q(x)-\bar{q})]\right) .
$$

Since the function $s$ is concave, the integrand here is maximised when $q(x)=\bar{q}$ and for small values of the fluctuating field $(q(x)-\bar{q})$ is quadratic in this variable. Hence since, as pointed out in Section $1, k$ is a microscopic quantity, extremely small on the macroscopic scale, it may be seen from the form of the exponent in Eq. (2.2.1) that $k^{1 / 2}$ is the natural normalisation factor for that field, since that exponent becomes $O(1)$ when $q(x)-\bar{q}=O\left(k^{1 / 2}\right)$. Accordingly, we represent the equilibrium fluctuations by the random field $\xi$, defined by the formula

$$
q(x)=\bar{q}+k^{1 / 2} \xi(x) .
$$

In accordance with general desiderata for field theories [SW], we assume that $\xi$ is a $\mathcal{D}^{\prime}(\Omega)^{n_{-}}$ class distribution, in the sense of L. Schwartz [Sc], and we represent its statistical properties by its characteristic function

$$
\mu_{\mathrm{eq}}(f)=E_{\mathrm{eq}}(\exp [i \xi(f)]) \forall f \in \mathcal{D}(\Omega)^{n},
$$

where $\mathcal{D}(\Omega)^{n}$, the dual of $\mathcal{D}^{\prime}(\Omega)^{n}$, is the space of smooth, $\mathbf{R}^{n}$ valued functions on $\Omega$ with support in that region, $E_{\text {eq }}$ is the expectation functional corresponding to the probability distribution $P$ and $\xi(f)$ is the smeared field obtained by integrating $\xi$ against the test function $f\left(=\left(f_{1}, \ldots, f_{n}\right)\right)$. We shall formulate $\mu_{\text {eq }}(f)$ in the limit $k \rightarrow 0$, which we term the Boltzmann limit. To this end, we note that, by Eq. (2.2.2), the exponent in Eq. (2.2.1) reduces to $\int_{\Omega} d x f(x) \cdot\left[s^{\prime \prime}(\bar{q}) f(x)\right] / 2$ in this limit. Hence, by Eq. $(2 . .2 .3), \mu_{\text {eq }}$ is given by the following formula in this limit.

$$
\mu_{\mathrm{eq}}(f)=\frac{\int D \xi \exp \left(\int_{\Omega} d x\left[\xi(x) \cdot s^{\prime \prime}(\bar{q}) \xi(x) / 2+i \xi(x) \cdot f(x)\right]\right)}{\int D \xi \exp \left(\int_{\Omega} d x\left[\xi(x) \cdot s^{\prime \prime}(\bar{q}) \xi(x)\right] / 2\right)},
$$


where $\int D \xi(x)$ denotes functional integration over the random field $\xi$. To provide a proper mathematical definition of this, we proceed as follows. Resolve $\Omega$ into a set of cells, $\Delta_{J}$, of equal volume, denote the values of $\xi$ and $f$ at the centroid of $\Delta_{J}$ by $\xi_{J}$ and $f_{J}$, respectively, and re-express Eq. (2.2.4) as

$$
\mu_{\mathrm{eq}}(f)=\lim _{\Delta} \Pi_{J}\left[\frac{\int_{R^{n}} d \xi_{J} \exp \left(\xi_{J} \cdot s^{\prime \prime}(\bar{q}) \xi_{J} / 2+i \xi_{J} f_{J}\right)}{\int_{R^{n}} d \xi_{J} \exp \left(\xi_{J} \cdot s^{\prime \prime}(\bar{q}) \xi_{J} / 2\right)}\right],
$$

where $\lim _{\Delta}$ signifies the limit in which the cells shrink to points. It then follows from Eqs. (2.1.12) and (2.2.5) that

$$
\mu_{\mathrm{eq}}(f)=\exp \left(-\frac{1}{2}\left(f, \pi^{\prime \prime}(\bar{\theta}) f\right)\right) \forall f \in \mathcal{D}(\Omega)^{n}
$$

In view of Eq. (2.2.3), this signifies that $\xi$ is a Gaussian random field with zero mean and two point fumction

$$
E_{e q}\left(\xi_{j}(x) \xi_{k}\left(x^{\prime}\right)\right)=\frac{\partial^{2} \pi(\bar{\theta})}{\partial \bar{\theta}_{j} \partial \bar{\theta}_{k}} \delta\left(x-x^{\prime}\right) \forall x, x^{\prime} \in \Omega, j, k=1,2 ., n
$$

In order to obtain the local properties of the random field $\xi$ in a global equilibrium state, we consider the action of $\mu_{\mathrm{eq}}$ on test functions that are strongly concentrated around an arbitrary point $x_{0}$ of $\Omega$. To this end we introduce the transformation $f \rightarrow f_{x_{0}, \epsilon}$ of $\mathcal{D}(\Omega)^{n}$, as defined by the formula

$$
f_{x_{0}, \epsilon}(x)=\epsilon^{-d / 2} f\left(\epsilon^{-1}\left(x-x_{0}\right)\right)
$$

where $\epsilon$ is a 'small' positive number. It follows immediately from this definition and Eq. (2.2.6) that $\mu_{\mathrm{eq}}$ is invariant under this transformation and therefore the local (punctual !) properties of this characteristic function are given by the equation

$$
\lim _{\epsilon \downarrow 0} \mu_{\mathrm{eq}}\left(f_{x_{0}, \epsilon}\right)=\exp \left(-\frac{1}{2}\left(f, \pi^{\prime \prime}(\bar{\theta}) f\right)\right) \forall f \in \mathcal{D}(\Omega)^{n}, x_{0} \in \Omega
$$

\subsection{The Quantum Mechanical Picture}

For its microscopic description, we employ the scaling for which the model occupies the spatial region $\Omega_{L}=L \Omega$ and we indicate its $L$ dependence by a subscript $L$ to $\Sigma$. In a standard way [VN], we represent its pure states and observables by the normalised vectors and the self-adjoint operators, respectively, in a separable Hilbert space, $\mathcal{H}_{L}$, canonically attached to the region $\Omega_{L}$. In particular, we denote the Hamiltonian of $\Sigma_{L}$ by $H_{L}$.

We formulate the statistical thermodynamics of $\Sigma_{L}$ in terms of a set of extensive,

conserved observables $\hat{Q}_{L}=\left(\hat{Q}_{1, L}=H_{L}, \hat{Q}_{2, L}, ., \hat{Q}_{n, L}\right)$. These are designed to be the 
quantum counterparts of the classical variables $Q$, and they intercommute, up to possible surface corrections.

The Von Neumann entropy density of a state represented by the density matrix $\rho_{L}$ is

$$
\hat{s}_{L}(\rho)=-L^{-d} k \operatorname{Tr}\left(\rho_{L} \log \rho_{L}\right)
$$

and the reduced pressure is given by the quantum mechanical version of Eq. (2.1.6), namely

$$
\begin{gathered}
\pi_{L}(\theta)=\sup _{\rho_{L}}\left(\hat{s}_{L}\left(\rho_{L}\right)-\theta \cdot \operatorname{Tr}\left(\rho_{L} \cdot \hat{Q}_{L}\right)\right)= \\
-\inf _{\rho_{L}} L^{-d} \operatorname{Tr}\left(k \rho_{L} \log \rho_{L}+\rho_{L} \cdot \hat{Q}_{L}\right) .
\end{gathered}
$$

Hence, as the infimum is attained when $\rho_{L}=\exp \left(-k^{-1} \theta \cdot \hat{Q}_{L}\right) / \operatorname{Tr}($ idem $)$,

$$
\pi_{L}(\theta)=L^{-d} \log \operatorname{Tr}\left(\exp \left(-k^{-1} \theta \cdot \hat{Q}_{L}\right)\right)
$$

Therefore, in the thermodynamic limit, the reduced pressure is given by the standard formula (cf. [Ru], [Ro1])

$$
\pi(\theta)=\lim _{L \rightarrow \infty} \ln \operatorname{Tr}\left(\exp \left(-k^{-1} \theta \cdot \hat{Q}_{L}\right)\right) .
$$

This formula provides the quantum mechanical infrastructure of the classical thermodynamical picture of Section 2.1.

We also formulate the microscopic structure of the equilibrium states of $\Sigma_{L}$ in the thermodynamic limit, wherein it is represented as an infinite system, $\Sigma_{\infty}$, which occupies the space $X$. We construct the model of $\Sigma_{\infty}$ in the following standard operator algebraic terms $[\mathrm{Ru}, \mathrm{Em}, \mathrm{Se} 1]$.

The Operator Algebraic Picture. We define $\mathcal{L}$ to be the set of bounded open connected regions, $\{\Lambda\}$, of $X$ and we denote the volume of $\Lambda$ by $|\Lambda|$. We represent the observables of $\Sigma_{\infty}$ that are located in the region $\Lambda(\in \mathcal{L})$ by the self-adjoint elements of a $W^{\star}$-algebra, $\mathcal{A}(\Lambda)$, operating in a separable Hilbert space $\mathcal{H}(\Lambda)$; and we assume that $\mathcal{A}(\Lambda)$ and $\mathcal{H}(\Lambda)$ satisfy the canonical demands of isotony and local commutativity, as given by the formulae

$$
\mathcal{H}\left(\Lambda_{1}\right) \subset \mathcal{H}\left(\Lambda_{2}\right) \text { and } \mathcal{A}\left(\Lambda_{1}\right) \subset \mathcal{A}\left(\Lambda_{2}\right) \text { if } \Lambda_{1} \subset \Lambda_{2}
$$

and

$$
\mathcal{A}\left(\Lambda_{1}\right) \subset \mathcal{A}\left(\Lambda_{2}\right)^{\prime} \text { if } \Lambda_{1} \cap \Lambda_{2}=\emptyset,
$$

respectively, $\mathcal{A}(\Lambda)^{\prime}$ being the commutant of $\mathcal{A}(\Lambda)$. We then define $\mathcal{A}_{\mathcal{L}}$ to be the normed *-algebra $\bigcup_{\Lambda \in \mathcal{L}} \mathcal{A}(\Lambda)$ and $\mathcal{A}$ to be $C^{\star}$-algebra given by its norm completion. These are termed the algebras of local and quasi-local bounded observables of $\Sigma_{\infty}$, respectively. The unbounded local observables of this system are represented by the unbounded self-adjoint affiliates of $\mathcal{A}_{\mathcal{L}}$ [Se4].

We assume that, for each $\Lambda \in \mathcal{L}$, the model has a set of extensive observables $\hat{Q}(\Lambda)(=$ $\left.\left(\hat{Q}_{1}(\Lambda), \hat{Q}_{2}(\Lambda), ., \hat{Q}_{n}(\Lambda)\right)\right)$ affiliated to $\mathcal{A}(\Lambda)$, which intercommute, up to possible surface 
corrections, and are the natural counterparts, for the region $\Lambda$, of the observables $\hat{Q}_{L}$ of $\Sigma_{L}$. In particular, $\hat{Q}_{1}(\Lambda)$ is the Hamiltonian of the system, $\Sigma(\Lambda)$, of particles of the given species confined to $\Lambda$.

We assume that the model is equipped with a representation $\sigma$ of the space translation group $X$ in $\operatorname{Aut}(\mathcal{A})$ and that $\mathcal{A}$ and $\hat{Q}$ transform covariantly under these translations, i.e. that

$$
\sigma(x) \mathcal{A}(\Lambda)=\mathcal{A}(\Lambda+x) \text { and } \sigma(x) \hat{Q}(\Lambda)=\hat{Q}(\Lambda+x) \forall x \in X, \Lambda \in \mathcal{L} .
$$

We take the states of $\Sigma_{\infty}$ to comprise the set, $\mathcal{S}$, of positive, normalised, linear functionals, $\phi$, on $\mathcal{A}$, whose restrictions to the local algebras $\mathcal{A}(\Lambda)$ are normal, in that they correspond to density matrices, $\rho_{\Lambda}^{\phi}$, in the Hilbert spaces $\mathcal{H}(\Lambda)$ according to the formula

$$
\phi(A)=\operatorname{Tr}\left(\rho_{\Lambda}^{\phi} A\right) \forall A \in \mathcal{A}(\Lambda), \Lambda \in \mathcal{L} .
$$

This latter condition of local normality is imposed in order to ensure that there is zero probability that a bounded spatial region can contain an infinite number of particles [DDR]. We represent the action of space translations on $\mathcal{S}$ by the dual of $\sigma(X)$, we denote by $\mathcal{S}_{X}$ the set of translationally invariant states of the system and by $\mathcal{S}_{X}^{(0)}$ its subset whose elements, $\phi$, admit well-defined expectation values of the global density of $\hat{Q}$ in the form

$$
\hat{q}(\phi)=\lim _{\Lambda \uparrow X} \frac{\operatorname{Tr}\left(\rho_{\Lambda}^{\phi} \hat{Q}(\Lambda)\right)}{|\Lambda|} .
$$

The functional $\hat{q}$ is manifestly affine. We assume that $\hat{q}(\phi)$ represents the value, for the state $\phi$, of the classical variable $q$ of Section 2.1 and thus that the range of $\hat{q}$ is just that of $q$, namely $\mathcal{Q}$.. The global entropy density functional, $\hat{s}$, on $\mathcal{S}_{X}$ is defined by the formula $[\mathrm{Ru}, \mathrm{Ro} 1]$

$$
\hat{s}(\phi)=\lim _{\Lambda \uparrow X} \frac{-k \operatorname{Tr}\left(\rho_{\Lambda}^{\phi} \ln \left(\rho_{\Lambda}^{\phi}\right)\right)}{|\Lambda|},
$$

and this functional is affine. We assume that its relationship to the classical entropy functional $s$ of Section 2.1 is given by the formula

$$
s(q)=\sup \{\hat{s}(\phi) \mid \hat{q}(\phi)=q\} .
$$

Hence the equilibrium condition (2.1.7) may be expressed in the form

$$
\pi(\theta)=\sup \{\hat{s}(\phi)-\theta \cdot \hat{q}(\phi) \mid \hat{q}(\phi)=q ; q \in \mathcal{Q}\}
$$

i.e.

$$
\pi(\theta)=\hat{s}(\phi)-\theta \cdot \hat{q}(\phi) .
$$

This is the GTS condition mentioned in Section 1. We denote by $\mathcal{G}_{\theta}$ the set of GTS states corresponding to the value $\theta$ of the control variable. 
Comment. The above derivation of this GTS condition was based on the classical thermodynamic formula (2.1.7) and the identification of $\hat{q}(\rho)$ and $\hat{s}(\phi)$ with $q$ and $s(q)$, respectively. The same formula has been obtained on purely quantum statistical grounds [Ro1] for lattice systems and for continuous systems of particles with hard cores, with $\hat{q}$ comprising the densities of energy and particle number.

Since both $\hat{s}$ and $\hat{q}$ are affine, it follows from Eq. (2.3.6) that $\mathcal{G}_{\theta}$ is convex. We define $\mathcal{E}\left(\mathcal{G}_{\theta}\right)$ to be the set of its extremals and assume that these are the pure thermodynamical phases corresponding to the value $\theta$ of the control variable.. This assumption is supported by the observation $[\mathrm{Ru}]$ that the extremals are just the GTS states for which the global density of $\hat{Q}$ is sharply defined, i.e. dispersionless. Further, by the argument of Section 2.1 leading from the convexity of $\pi$ to the identification of $-q$ with the tangents $\mathcal{T}_{\theta}$, we see from Eq. (2.3.6) that $\mathcal{G}_{\theta}=\left\{\phi \in \mathcal{S}_{X}^{(0)} \mid \hat{q}(\phi) \in-\mathcal{T}_{\theta}\right\}$.

Thermodynamical Completeness Conditions. We take these conditions, as applied to the thermodynamical functional $\hat{q}\left(=\left(\hat{q}_{1}, . ., \hat{q}_{n}\right)\right)$, and thus to the classical variables $q=\left(q_{1}, \ldots, q_{n}\right)$, to be that

(i) $\hat{q}$ separates the extremals of $\mathcal{G}_{\theta}$, i.e. if $\phi_{1}$ and $\phi_{2}$ are different elements of $\mathcal{E}\left(\mathcal{G}_{\theta}\right)$, then $\hat{q}\left(\phi_{1}\right) \neq \hat{q}\left(\phi_{2}\right)$; and

(ii) no proper subset of $\left(\hat{q}_{1}, \ldots, \hat{q}_{n}\right)$ separates all the extremals of $\mathcal{G}_{\theta}$.

These conditions signify that, for $q \in \mathcal{Q}$, there is a unique extremal GTS state for which the value of $\hat{q}$ is $q$. We denote this state by $\psi_{q}$. In the pure phase regime, where $q=-\pi^{\prime}(\theta)$, we denote it, equivalently, by $\omega_{\theta}$.

Dynamics and the KMS Conditions. Since, by Eqs. (2.1.1) and (2.1.5), the sum of the thermal and mechanical energies, $T d S$ and $-p d V$, required to produce an increment $d Q$ in the classical variable $Q$ is $d E+\sum_{j=2}^{n} f_{j} d Q_{j}=\theta_{1}^{-1} \theta \cdot d Q$, we assume that, correspondingly, the effective Hamiltonian for the finite version, $\Sigma(\Lambda)$, of $\Sigma$ is

$$
H_{\theta}^{\Lambda}=\theta_{1}^{-1} \theta \cdot \hat{Q}(\Lambda)
$$

Hence the dynamics of $\Sigma(\Lambda)$ is governed by the one parameter group of automorphisms $\left\{\alpha_{\theta}^{\Lambda}(\tau) \mid \tau \in \mathbf{R}\right\}$ of $\mathcal{A}(\Lambda)$ given by the formula

$$
\alpha_{\theta}^{\Lambda}(\tau) A=\exp \left(i H_{\theta}^{\Lambda} \tau / \hbar\right) A \exp \left(-i H_{\theta}^{\Lambda} \tau / \hbar\right) .
$$

Since these are inner automorphisms they extend to the whole algebra $\mathcal{A}$.

We now recall that although, in the case of lattice systems, the dynamics of $\Sigma_{\infty}$ may be formulated in terms of automorphisms of $\mathcal{A}$ that are norm limits of $\alpha_{\theta}^{\Lambda}$ [St, Ro2], the same is not generally true for continuous systems [DS, Ra]. For these, it is only certain privileged representations of $\mathcal{A}$ that support a dynamics that must be based on a weaker limiting form of that of $\Sigma(\Lambda)$ as $\Lambda$ increases to $X$ [Se5]. Accordingly, we employ the following scheme to formulate the dynamics of $\Sigma_{\infty}$ in such representations.

We denote by $\left(\mathcal{H}_{\phi}, R_{\phi}, \Psi_{\phi}\right)$ the GNS triple of a state $\phi$. Then its normal folium $\mathcal{F}(\phi)$, which comprises the states corresponding to the density matrices in $\mathcal{H}_{\phi}$, is the predual 
of the $W^{\star}$-algebra $R_{\phi}(\mathcal{A})^{\prime \prime}$. We term this folium dynamically amenable relative to the control parameter $\theta$ if it supports a dynamics given by a one parameter group of affine transformations $\left\{\hat{\alpha}_{\star \theta}(t) \mid t \in \mathbf{R}\right\}$ of $\mathcal{F}(\phi)$ given by the $w^{\star}$ limit, as $\Lambda$ increases to $X$, of the predual of the automorphisms $\alpha_{\theta}^{\Lambda}$. Thus

$$
\left\langle\hat{\alpha}_{\star \theta}(t) \phi^{\prime} ; A\right\rangle=\lim _{\Lambda \uparrow X}\left\langle\phi^{\prime} ; \alpha_{\theta}^{\Lambda}(t) A\right\rangle \forall \phi^{\prime} \in \mathcal{F}(\phi), A \in \mathcal{A}, t \in \mathbf{R} .
$$

The dual of the transformations $\hat{\alpha}_{\star \theta}$ is then a one parameter group $\left\{\hat{\alpha}_{\theta}(t) \mid t \in \mathbf{R}\right\}$ of automorphisms of $R_{\phi}(\mathcal{A})^{\prime \prime}$ [Se5]. We denote by $\hat{\phi}$ the canonical extension of $\phi$ to this algebra.

The KMS conditions on the state $\phi$ are that it is dynamically amenable and that

$$
\hat{\phi}\left(\left[\hat{\alpha}_{\theta}(\tau) \hat{A}\right] \hat{B}\right)=\hat{\phi}\left(\hat{B} \hat{\alpha}_{\theta}(\tau+i \hbar \beta) \hat{A}\right) \forall \hat{A}, \hat{B} \in R_{\phi}(\mathcal{A})^{\prime \prime}, t \in \mathbf{R},
$$

where $\beta=(k T)^{-1}=\theta_{1} / k$.

As first proposed by $[\mathrm{HHW}]$, these conditions are designed to characterise the equilibrium states of the system, and this proposal is supported by treatments of their dynamic [HKT-P] and thermodynamic [PW, Se6] stability. In Section 4 we shall assume that the GTS states satisfy these conditions, even though the available proofs are confined to lattice systems and a certain class of continuous ones (cf. the discussion in [Se1, P.122]).

\section{Nonequilibrium Thermodynamics and Local Equilibrium.}

In the previous Section, we saw that, at each level of macroscopicality, the equilibrium states are labelled by the value of the classical thermodynamical variable $q$. For a nonequilibrium state, we now take our LTE condition to be that, at each level, the local form of the state is the same as for equilibrium, but with that variable now taking the space-time dependent form $q(x, t)$ governed by the macroscopic evolution of the system. Our objective is to pass from the descriptions of global to local equilibrium, at three levels of macroscopicality, in nonequilibrium situations.

\subsection{The Macroscopic Picture.}

The phenomenological law (1.1) is generally based on the following assumptions [DM, LL].

(i) The density $q$ of $Q$ is a function of position $x$ and time $t$ that satisfies a local conservation law,

$$
\frac{\partial}{\partial t} q(x, t)+\nabla \cdot j(x, t)=0
$$

where $j=\left(j_{1}, \ldots, j_{n}\right)$ is the associated current density.

(ii) The model $\Sigma$ supports a thermodynamics in which the local entropy density is the equilibrium entropy function, $s$, of $q(x, t)$. This is the local equilibrium assumption. It serves to define the local control variable, $\theta(x, t)$, conjugate to $q(x, t)$ by the canonical counterpart of Eq. (2.1.4), namely

$$
\theta(x, t)=s^{\prime}(q(x, t))
$$


Moreover, the total, time-dependent entropy is

$$
S(t)=\int_{\Omega} d x s(q(x, t))
$$

(iii) The current $j(x, t)$ is determined by the control variable $\theta(x, t)$ according to a constitutive equation of the form

$$
j(x, t)=\mathcal{F}(\theta ; x, t),
$$

which, together with Eqs. (3.1.1) and (3.1.2), leads to a positive entropy production rate, in accordance with the second law. Moreover, by Eq. (3.1.2), this formula for the current may be expressed as

$$
j(x, t)=\mathcal{J}(q ; x, t):=\mathcal{F}\left(s^{\prime}(q) ; x, t\right),
$$

and consequently, by Eqs. (3.1.1) and (3.1.5), $q$ evolves autonomously according to the equation

$$
\frac{\partial}{\partial t} q(x, t)+\nabla \cdot \mathcal{J}(q ; x, t)=0
$$

which is equivalent to Eq. (1.1), with $\Phi(q ; x, t)=-\nabla \cdot \mathcal{J}(q ; x, t)$.

We represent the local thermodynamic state of $\Sigma$ by $q(x, t)$, or equivalently, in the pure phase regime, by $\theta(x, t)$. In fact we shall henceforth restrict our considerations to this regime since, as observed in Section 2.3, the underlying quantum statistics admits sharp definition of $q$ in pure phases only

We remark that Eqs. (2.1.5) and (3.1.2) suggest that $\theta_{1}(x, t)$ is just the reciprocal of a local temperature $T(x, t)$ : we leave until Section 4 a quantum statistical justification of this interpretation that is related to the zeroth law.

To summarise, under the above assumptions (i)-(iii), the macroscopic dynamics of the model is given by an autnomous law of the form represented by Eq. (1.1), with boundary conditions determined by the reservoirs $\mathcal{R}$. Specifically, we assume that the value of $\theta$ at a point of the boundary of $\Omega$ is equal to that of the corresponding control variable of the reservoir that is in contact with $\Sigma$ there. In particular, we take the equilibrium states of the continuum model to be the solutions of Eq. (1.1) for which both $q$ and the imposed boundary values of $\theta$ are stationary and spatially uniform.

\subsection{Hydrodynamical Fluctuations: the Mesoscopic Picture}

We represent the hydrodynamical fluctuations about the deterministic flow $q(x, t)$ by a random field $\xi_{t}(x)$, normalised, as in the equilibrium situation, by the factor $k^{1 / 2}$. Thus, the local density of $Q$ becomes $q(x, t)+k^{1 / 2} \xi_{t}(x)$, which is the natural generalisation of the formula for equilibrium fluctuations given by Eq. (2.2.2). As in Section 2.2, we take the random field $\xi_{t}$ to be a $\mathcal{D}^{\prime}(\Omega)^{n}$ class distribution. Its statistical properties are represented by its characteristic function

$$
\mu(f ; t)=E\left(\exp \left(i \xi_{t}(f)\right)\right) \forall f \in \mathcal{D}(\Omega)^{n},
$$


where $E$ is its expectation functional and $\xi_{t}(f)$ is the smeared field obtained by integrating $\xi_{t}$ against $f$. We now assume that, for each time $t$, the local properties of $\mu$ are just those of $\mu_{\mathrm{eq}}$, as given by Eq. (2.2.9), but with $\bar{\theta}$ replaced by $\theta(x, t)$. Thus our local equilibrium condition for the fluctuating field is that

$$
\lim _{\epsilon \downarrow} \mu\left(f_{x, \epsilon} ; t\right)=\mu_{\theta(x, t)}(f)
$$

where $f_{x, \epsilon}$ is defined by Eq. (2.2.8) and

$$
\mu_{\theta(x, t)}(f)=\exp \left[-\frac{1}{2}\left(f, \pi^{\prime \prime}(\theta(x, t)) f\right)\right] \forall f \in \mathcal{D}(\Omega)^{n}, x \in \Omega
$$

We represent the local state of the model at the mesoscopic level by this characteristic function

To make the LTE condition perhaps more transparent, we note that, by Eq. (2.2.8),

$$
\xi_{t}\left(f_{x, \epsilon}\right)=\xi_{t, x, \epsilon}(f)
$$

where

$$
\xi_{t, x, \epsilon}\left(x^{\prime}\right)=\epsilon^{d / 2} \xi_{t}\left(x+\epsilon x^{\prime}\right) .
$$

Hence, by Eqs. (3.2.1), (3.23) and (3.2.5), the formula (3.2.2) may be expressed in the form

$$
\lim _{\epsilon \downarrow 0} E\left[\exp \left(i \xi_{t, x, \epsilon}(f)\right)\right]=\exp \left(\frac{1}{2}\left(f, \pi^{\prime \prime}(\theta(x, t)) f\right)\right) \forall f \in \mathcal{D}(\Omega)^{n}, x \in \Omega, t \in \mathbf{R}
$$

This signifies that, in the punctual limit where $\epsilon$ tends to zero, $\xi_{t, x, \epsilon}$ becomes a Gaussian random field with zero mean and covariance $\pi^{\prime \prime}(\theta(x, t))$

\subsection{The Quantum Mechanical Picture.}

We consider the state of $\Sigma_{L}$ in a region that, in the macroscopic scaling, is an open neighbourhood, $\mathcal{N}(\epsilon, x)$, of an arbitrary point $x$ of $\Omega$, where $\epsilon$ is a real positive valued parameter and $\mathcal{N}(\epsilon, x)$ shrinks to the point $x$ as $\epsilon \rightarrow 0$. Then the region $\mathcal{N}(\epsilon, x)$ of the macroscopic picture is just the neighbourhood $\mathcal{N}_{L}(\epsilon, x):=L \mathcal{N}(\epsilon, x)$ of $L x$ for the microscopic one. Thus, for small fixed $\epsilon$ and sufficiently large $L$, this region is small from the macroscopic standpoint yet large from the microscopic one, indeed sufficiently large to contain enormous numbers of particles. It therefore corresponds to a hydrodynamic point in the limit where first $L \rightarrow \infty$ and then $\epsilon \rightarrow 0$.

We denote by $\tilde{\Omega}_{L}$ and $\tilde{\mathcal{N}}_{L}(\epsilon, x)$ the regions $\Omega_{L}$ and $\mathcal{N}_{L}(\epsilon, x)$, respectively, as viewed relative to the point $L x$ of $\Omega_{L}$. Thus $\tilde{\Omega}_{L}:=\Omega_{L}-L x$ and $\tilde{\mathcal{N}}_{L}(\epsilon, x):=\mathcal{N}_{L}(\epsilon, x)-L x$. It follows from these specifications that this latter region is infinitely distant from the boundary $\partial \tilde{\Omega}_{L}$ of $\tilde{\Omega}_{L}$ in the limit $L \rightarrow \infty$; and moreover that, in this limit, it covers the whole space $X$ 
The algebra of observables for the region $\tilde{\mathcal{N}}_{L}(\epsilon, x)$ is just the union of the local algebras $\mathcal{A}(\Lambda)$ of its subregions $\Lambda$ and this reduces to $\mathcal{A}_{\mathcal{L}}$ in the limit $L \rightarrow \infty$. Accordingly, we assume that the local equilibrium condition for a pure phase in $\tilde{\mathcal{N}}_{L}(\epsilon, x)$ is that of GTS corresponding to the prevailing value of $\theta(x, t)$. Specifically, denoting by $\phi_{L, t}$ the state of $\Sigma_{L}$ at time $t$ and by $\phi_{L, t, x, \epsilon}$ its restriction to the region $\tilde{\mathcal{N}}_{L}(\epsilon, x)$, we take the local equilibrium condition to be that

$$
\lim _{\epsilon \rightarrow 0} \lim _{L \rightarrow \infty} \phi_{L, t, \epsilon, x}(A)=\omega_{\theta(x, t)}(A) \forall A \in \mathcal{A}_{L} .
$$

where $\omega_{\theta}$ is the GTS state of $\Sigma_{\infty}$ corresponding to the value $\theta$ of the control variable in a pure phase, as specified in Section 2.3. Thus, reverting to the macroscopic scaling, we represent the LTE assumption by the attachment of a GTS state $\omega_{\theta(x, t)}$ to the point $x$ of $\Omega$.

Fibre Bundle Picture. The situation that we have just decribed may be simply represented in terms of the fibre bundle $\mathcal{B}:=\Omega_{\mathbf{R}} \times \mathcal{A}$, where $\Omega_{\mathbf{R}}:=\Omega \times \mathbf{R}$. (cf. [SS]). There, the state $\Phi$ of $\mathcal{B}$ corresponding to the space-time profile $\theta(x, t)$ of the macroscopic field $\theta$ is given by the formula

$$
\Phi((x, t), A)=\omega_{\theta(x, t)}(A) \forall x \in \Omega, t \in \mathbf{R}, A \in \mathcal{A} .
$$

Evidently the fibre bundle description provides a macroscopic-cum-microscopic picture of local equilibrium.

Local Microdynamics and Local KMS Condition. We assume here that, as envisaged in Section 1, the time scales for the macroscopic and microscopic dynamics are infinitely separated. Thus the local macroscopic variable $q(x, t)$ is effectively 'frozen' at a fixed value and the condition for LTE is that the state of the system in a local submacroscopic region is the corresponding equilibrium state.

We now supplement the LTE assumption for the region $\tilde{\mathcal{N}}_{L}(\epsilon, x)$ by a formulation of the microscopic dynamics of the model there. For this, we assume that the interactions of the model are of short range. Thus, taking account of the facts that, in the limit $L \rightarrow \infty$, the region $\tilde{\mathcal{N}}_{L}(\epsilon, x)$ covers the whole space $X$ and that its algebra of observables is $\mathcal{A}_{\mathcal{L}}$, we assume that its microscopic dynamics in the normal folium of the local equilibrium state $\omega_{\theta(x, t)}$ takes the same form as for that in the GTS state $\omega_{\theta}$ of Section 2.3, with $\theta$ replaced by $\theta(x, t)$. On this basis, we assume that, in the limit where first $L \rightarrow \infty$ and then $\epsilon \rightarrow 0$, the microscopic dynamics in $\tilde{\mathcal{N}}_{L}(\epsilon, x)$ is given by the one- parameter grouip $\left\{\hat{\alpha}_{\theta(x, t)}(\tau) \mid \tau \in \mathbf{R}\right\}$ of automorphisms of $R_{\omega_{\theta(x, t)}}(\mathcal{A})^{\prime \prime}$, as defined in Section 2.3, with $R_{\omega_{\theta(x, t)}}$ the GNS representation of $\mathcal{A}$ for the state $\omega_{\theta(x, t)}$.

Correspondingly we assume that, on grounds discussed in Section 2.3, the GTS state $\omega_{\theta(x, t)}$ satisfies the KMS condition corresponding to the prevailing value, $\theta(x, t)$, of the control parameter. This condition is given by the canonical analogue of Eq. (2.3.10), namely

$$
\hat{\omega}_{\theta(x, t)}\left(\left[\hat{\alpha}_{\theta(x, t)}(\tau) \hat{A}\right] \hat{B}\right)=\hat{\omega}_{\theta(x, t)}\left(\hat{B}\left[\hat{\alpha}_{\theta(x, t)}(\tau+i \beta) \hat{A}\right]\right) \forall \hat{A}, \hat{B} \in R_{\omega_{\theta(x, t)}}(\mathcal{A})^{\prime \prime}, \tau \in \mathbf{R},
$$




$$
(x, t) \in \Omega_{\mathbf{R}},
$$

where $\beta=(k T(x, t))^{-1}=\theta_{1}(x, t) / k$. This is the local KMS condition.

\section{Local Zeroth Law.}

Suppose now that $\Sigma$ is coupled to a finite system, $\Gamma$, the interaction being located within a region which, in the microscopic picture, is a bounded, $L$-independent neighbourhood, $\Delta$, of the point $L x$ of $\Omega_{L}$. Thus, for $L$ sufficiently large, $\Delta$ is contained in the neighbourhood $\tilde{\mathcal{N}}_{L}(\epsilon, x)$ of $L x$ : moreover, in the macroscopic picture, it reduces to the point $x$ in the limit where $\epsilon \rightarrow 0$.

We now assume the local KMS condition (3.3.3), and recall that the coupling of a finite system to an infinite one in a KMS state serves to drive the former to equilibrium at the temperature of the latter [KFGV]. It follows that the local $\Sigma-\Gamma$ interaction will drive $\Gamma$ to equilibrium at temperature $T(x, t)$. Thus, under the assumption of local equilibrium, we have a local version of the zeroth law

\section{Concluding Remarks}

We have provided mathematical specifications of local thermodynamic equilibrium (LTE) at macroscopic, mesoscopic and microscopic levels within the framework of nonrelativistic quantum statistical thermodynamics. Furthermore, we provide a precise condition (in Section 2.3) for the thermodynamic completeness of $Q$.

The coordination of our LTE conditions for the single phase regime at the three different levels serves to express them all in terms of $\theta(x, t)$. Specifically, it represents the local state of $\Sigma$ by $\theta(x, t)$ at the macroscopic level, by the characteristic function $\mu_{\theta(x, t)}$ at the mesoscopic one (cf. Eq. (3.2.3)) and the quantum mechanical form $\omega_{\theta(x, t)}$ at the microscopic level. Furthermore, by considering the local coupling of $\Sigma$ to a finite one, $\Gamma$, we showed that the LTE condition implied a local version of the zeroth law.

Our derivation of these results was dependent on the assumption of scale invariance of the phenomenological dynamics. This permitted the employment of a hydrodynamical limit, which carried an infinite separation of the macroscopic and microscopic time scales. However, as noted in Section 2.2, the scale invariance assumption is not satisfied in the all-important case of Navier-Stokes fluid mechanics, though the ratio of the macroscopic to microscopic time scales is still enormously large there. In this and similar cases one may employ the methods of [Se3] to obtain the results of the present work, up to miniscule corrections of the order of ratios of microscopic to corresponding macroscopic quantities.

Finally we remark that, while the present work is designed to be a contribution to condensed matter physics, a conceptually different treatment of local equilibrium has been formulated in $[\mathrm{BOR}]$ within the framework of quantum field theory.

\section{References}


[BOR] D. Buchholtz, I. Ojima and H. Roos: Ann. Phys. 297 (2002), 219

[Ca] H. B. Callen: Thermodynamics and an Introduction to Thermostatistics, Wiley, New York, 1985

[Da] E. B. Davies: J. Stat. Phys. 18 (1978), 161.

[DDR], G.-F. Dell'Antonio, S. Doplicher and D. Ruelle: Commun. Math. Phys. 2 (1966), 223

[DIPP] A. De Masi, N. Ianiro, A. Pellegrinotti and E. Presutti: From Sochstics to Hydrodynamics, Pp. 127-294 of Nonequilibrium Phenomena II, Ed. J. L. Lebowitz and E. W. Montroll, 1984

[DM] S. R. De Groot and P. Mazur: Nonequilibrium Thermodynamics, North Holland, Amsterdam, 1962

[DS] D. A. Dubin and G. L. Sewell: J. Math. Phys. 11 (1970), 2990

[Em] G. G. Emch: Algebraic Methods in Statistical Mechanics and Quantum Field Theory, Wiley, New York, 1972

[GVV] D. Goderis, A. Verbeure and P. Vets: Prob. Th. Rel. Fields 82, 527, 1989

[HHW] R. Haag, N. M. Hugenholtz and M. Winnink: Commun. Math. Phys. 5 (1967), 215

[HKT-P] R. Haag, D. Kastler and E. B. Trych-Pohlmeyer: Commun. Math. Phys. 38 (1974), 173

[KFGV] A. Kossakowski, A. Frigerio, V. Gorini and M. Verri: Commun. Math. Phys. 57 (1977), 97

[KMP] C. Kipnis, C. Marchioro and E. Presutti: J. Stat. Phys. 27 (1982), 85

[LL] L. D. Landau and E. M. Lifschitz: Fluid Mechanics, Pergamon, Oxford, 1984

[NY] B. Nachtergaele and H-T Yau: Commun. Math. Phys. 243 (2003), 485

[PW] W. Pusz and S. L. Woronowicz: Commun. Math. Phys. 58 (1978), 273

[Pr] E. Presutti: Scaling Limits in Statistical Mechanics and Microstructures in Continuum Mechanics, Springer, Berlin, Heidelberg, 2009

[Ra] C. Radin: Commun. Math. Phys. 54 (1977), 69

[Ro1] D. W. Robinson: The Thermodynamic Pressure in Quantum Statistical Mechanics: Lecture Notes in Physics Vol. 9, Springer, Berlin, 1971

[Ro2] D. W. Robinson: Commun. Math. Phys. 7 (1968), 337

[Ru] D. Ruelle: Statistical Mechanics, Benjamin, New York, 1969

[Sc] L. Schwartz: Theorie des Distributions, Hermann, Paris, 1998 
[Se1] G. L. Sewell: Quantum Mechanics and its Emergent Macrophysics, Princeton Univ. Press, Princeton, Oxford, 2002

[Se2] G. L. Sewell: Rev. Math. Phys. 17 (2005), 977

[Se3] G. L. Sewell: Rep. Math. Phys. 70 (2012), 251

[Se4] G. 1. Sewell: J. Math Phys. 11 (1970), 1968

[Se5] G. L. Sewell: Lett. Math. Phys. 6 (1982), 209

[Se6] G. L. Sewell: Phys. Rep. 57 (1980), 307

[SS] R. N. Sen and G. L. Sewell: J. Math. Phys. 43 (2002), 1323

[St] R. F. Streater: Commun. Math. Phys. 6 (1967), 233

[SW] R. F. Streater and A. S. Wightman: PCT, Spin and Statistics and All That, Benjamin, New York, 1964

[VN] J. Von Neumann: Mathematical Foundations of Quantum Mechanics, Princeton Univ. Press, Princeton, 1955 